\title{
Kava root extracts hinder prostate cancer development and tumorigenesis by involvement of dual inhibition of MAO-A and LSD1
}

\author{
Xuesen $\mathrm{Li}^{1}$, Liankun Song ${ }^{1}$, Shan $\mathrm{Xu}^{1}$, Matthew Tippin ${ }^{1}$, Shuan Meng ${ }^{1}$, Jun Xie ${ }^{1}$, Edward Uchio ${ }^{1,2}$, Xiaolin \\ $\mathbf{Z i} \mathbf{i}^{1,2,3}$ \\ 'Department of Urology, University of California, Irvine, Orang, CA 92868, USA. \\ ${ }^{2}$ Chao Family Comprehensive Cancer Center, University of California, Orange, CA 92868, USA. \\ ${ }^{3}$ Department of Pharmaceutical Sciences, University of California, Irvine, Irvine, CA 92617, USA.
}

Correspondence to: Prof. Xiaolin Zi, Department of Urology, University of California, Irvine, 101 The City Drive South, Rt.81 Bldg.55 Rm.204, Orange, CA 92868, USA. E-mail: xzi@uci.edu

\begin{abstract}
How to cite this article: Li X, Song L, Xu S, Tippin M, Meng S, Xie J, Uchio E, Zi X. Kava root extracts hinder prostate cancer development and tumorigenesis by involvement of dual inhibition of MAO-A and LSD1. J Trans/ Genet Genom 2021;5:163-72. https://dx.doi.org/10.20517/jtgg.2021.22
\end{abstract}

Received: 14 Apr 2021 First Decision: 14 May 2021 Revised: 16 May 2021 Accepted: 25 May 2021 First online: 28 May 2021

Academic Editor: Sanjay Gupta Copy Editor: Xi-Jun Chen Production Editor: Xi-Jun Chen

\begin{abstract}
Aim: Here, we aim to evaluate the chemopreventive efficacy of kava root extracts (KRE) in transgenic adenocarcinoma of the mouse prostate (TRAMP) mice and investigate potential molecular targets of kavalactones, the main components of kava.

Methods: TRAMP mice were administrated with KRE formulated food for different periods of time, and then the incidences of high-grade prostatic intraepithelial neoplasia (HG-PIN) and adenocarcinomas and tumor burdens were compared between vehicle control and KRE food fed groups. In addition, the inhibitory effect of the KRE and kavalactones on monoamine oxidase A (MAO-A) and lysine-specific demethylase 1 (LSD1) enzyme activities were examined by commercially available inhibitor screening kits. Histone $\mathrm{H} 3$ lysine 9 dimethylation was also evaluated in prostate cancer cells and tumor tissues using Western blotting analysis.
\end{abstract}

Results: Dietary feeding of $0.3 \%$ and $0.6 \%$ KRE to TRAMP mice from ages of 6 weeks to 12 weeks inhibited HGPIN by $43.5 \%$ and $59.7 \%$, respectively, and prostate adenocarcinoma by $53.5 \%$ and $66.4 \%$, respectively. In addition, $0.6 \%$ KRE fed TRAMP mice from ages of 6 weeks to 24 weeks exhibited a significant reduction of genitourinary weight (a surrogate of tumor burden) by $54.5 \%$ and reduced body weight gain. Furthermore, the KRE 
and kavalactones showed a significant inhibition of LSD1 and MAO-A enzyme activities.

Conclusion: Our results suggest that consumption of kava products through diet can delay prostate cancer development and progression and that kavalactones may be a new structure model for developing a potent dual inhibitor of LSD1 and MAO-A.

Keywords: Kava, chemoprevention, LSD1, MAO-A

\section{INTRODUCTION}

Prostate cancer has a projected incidence of $\sim 248,530$ new cases diagnosed, accounting for $\sim 34,130$ deaths in 2021 in the United States, ranking the second leading cause of cancer death in $\operatorname{men}^{[1]}$. The majority of men diagnosed with prostate cancer (about 4 in 5 men) die from other reasons rather than prostate cancer. More than 3.1 million men are alive with a diagnosis of prostate cancer in the United States ${ }^{[1]}$. In addition, over one-third of prostate cancer is slowly growing or progressing over several decades. This group of patients without cancer-related symptoms do not warrant aggressive, immediate treatment ${ }^{[2]}$. Instead, they are closely monitored for their indolent status using prostate-specific antigen (PSA) kinetics, periodic biopsies for histologic progression, and possibly surveillance magnetic resonance imaging (MRI) until further treatment is needed as judged by physicians. This emergent strategy for the management of indolent prostate cancer is called active surveillance ${ }^{[2]}$. Men on active surveillance have high anxiety, depression and doubts about the possible progression of the disease and are highly motivated to prevention approaches using dietary or pharmacological means or positive lifestyle changes ${ }^{[3]}$. Therefore, men on active surveillance have been thought to be an ideal population for chemo/dietary prevention methods to further reduce disease progression and anxiety during the period of active surveillance. Currently very few chemopreventive agents are effective for their testing in chemoprevention studies in this population.

Kava (Piper methysticum Forst) is a continually regrowing shrub native to the South Pacific Islands ${ }^{[4]}$. Traditionally, kava root extracts (KRE) have been prepared with coconut milk and water as a drink for a social gathering in the Pacific Islands for thousands of years ${ }^{[4]}$. The kava drink improves sleep quality and produces distinct muscle relaxation without drug addiction ${ }^{[4]}$. Several clinical studies have shown commercially available KRE to reduce anxiety and sold as an anxiolytic agent for several decades ${ }^{[5-7]}$. Kava drinking was also linked to lower cancer risk by an epidemiological study ${ }^{[8]}$. In addition, KRE has demonstrated potent anti-carcinogenic activity in several cancers, including bladder cancer, lung cancer, and others ${ }^{[9-12]}$. Intriguingly, compared to other areas of the world, incidences of prostate cancer in kava drinking countries, such as Fiji, are very low. However, when Fijian men moved to Australia, their prostate cancer incidence raised by 5.1 -fold ${ }^{[13,14]}$. These results have led us to investigate whether kava consumption can affect prostate cancer development and progression. We have previously shown that oral administration of KRE through dietary supplementation effectively reduced the growth of patient-derived xenograft (PDX) tumors and down-regulated the protein levels of androgen receptor (AR) and the expression of AR target genes PSA and transmembrane protease, serine 2 (TMPRESS 2$)^{[\rho]}$. Given the above-described properties of KRE, KRE would have the promise to be further studied as a chemopreventive agent for reducing progression risk and anxiety in prostate cancer patients under active surveillance.

To evaluate the chemopreventive effect of $K R E$, transgenic adenocarcinoma of the mouse prostate (TRAMP) mice were administrated with vehicle control or KRE formulated food in different stages of tumor development to test its ability to prevent prostate cancer development [i.e., occurrence of high-grade prostatic intraepithelial neoplasia (HG-PIN) and prostate adenocarcinomas] or delay progression. In addition, the effects of the KRE and its main components of kavalactones, including kawain, 
dihydrokawain, yangonin, and methysticin, on monoamine oxidase A (MAO-A) and lysine-specific demethylase 1 (LSD1) enzyme activities were examined. Our results have shown that the KRE significantly reduced the occurrences of HG-PIN and prostate adenocarcinomas and slowed tumor growth in the TRAMP transgenic mice, and inhibited both MAO-A and LSD1 activities in prostate cancer cells.

\section{METHODS}

\section{Study materials}

Authenticated LNCaP and $\mathrm{C} 4-2 \mathrm{~B}$ cell lines without mycoplasma contamination were used as described in details in our previous publication ${ }^{[15]}$. The KRE $(150 \mathrm{mg} / \mathrm{mL}$ kavalactones in $50 \%$ ethanol) was purchased from Gaia Herbs (Brevard, NC). Main kavalactones, including kawain, 5',6'-dehydrokawain, yangonin, and methysticin, were isolated and purified by LKT Laboratories, Inc. (St. Paul, MN) from the KRE. Antibodies against MAO-A and tubulin were from Santa Cruz Biotechnology, Inc. (Santa Cruz, CA). Di-methylated histone $\mathrm{H} 3$ lysine 4 (H3K4) and $\mathrm{H}_{3} \mathrm{~K} 9$ and $\mathrm{Ki} 67$ antibodies were purchased from Abcam (Cambridge, MA). MAO-A and LSD1 activity assay kits were from Cayman Chemical Company (Ann Arbor, Michigan).

\section{Prevention and intervention protocols in TRAMP mice}

A cohort of male hemizygous TRAMP mice was obtained by breeding female hemizygous C57BL/TGN TRAMP mice with male FVB/N mice and genotyped by a polymerase chain reaction (PCR) method as described in details in our previous publication ${ }^{[16]}$. The $0.3 \%(3 \mathrm{~g} / \mathrm{kg}$ food) or $0.6 \%(6 \mathrm{~g} / \mathrm{kg}$ food) KRE containing rodent food was formulated into AIN-93M rodent food by Dyets, Inc. through customer services. University of California, Irvine approved protocol (\#2007-2740) was followed for animal care and treatments. For the prevention protocol, $0.3 \%$ or $0.6 \%$ KRE containing food or vehicle control food was given to TRAMP mice from ages of 6 weeks to 12 weeks. For the intervention protocol, $0.3 \%$ or $0.6 \%$ KRE containing food or vehicle control food was administrated to 6 weeks old TRAMP mice until they were at the age of 24 weeks old.

The body weight and food consumption were recorded weekly until the end of the experiments. Organ weights, including liver, spleen, kidney, etc. and genitourinary (GU) weights were also measured at the end of the experiments and fixed in formalin for standard H\&E slide preparation and examination ${ }^{[16]}$. PIN lesions and prostate adenocarcinoma were evaluated to Dr. Cardiff's description ${ }^{[17]}$ and our previous publication $^{[16]}$.

\section{LSD1 inhibition assay ${ }^{[18]}$}

LSD1 inhibitor screening kit was purchased from Cayman Chemicals with the catalog number 700120. Human recombinant LSD1 enzymes are incubated with a selected concentration of compounds or with DMSO for $10 \mathrm{~min}$. Then a premix of reaction buffers solution that contains fluorescence substrate and horseradish peroxide (HRP) was added to the LSD1 solution. Finally, methylated peptides, which are the first 21 amino acids of the N-terminal tail of methylated H3K4, were added to the LSD1-buffer solution to begin the enzymatic processing. This process took place at $37{ }^{\circ} \mathrm{C}$ for $60 \mathrm{~min}$. In the process of demethylation, hydrogen peroxide $\left(\mathrm{H}_{2} \mathrm{O}_{2}\right)$ is formed as a byproduct. $\mathrm{HRP}$ then can use $\mathrm{H}_{2} \mathrm{O}_{2}$ to convert the non-fluorescence substrate resazurin into the fluorescence substrate resorufin, which was measured by a fluorescence spectrophotometer using the excitation value of $530 \mathrm{~nm}$ and the emission value of $585 \mathrm{~nm}$.

\section{MAO-A inhibition assay ${ }^{[19-21]}$}

MAO-A activity assay kit was purchased from Cayman Chemicals. The measurement of MAO-A activity in vehicle control $(0.1 \%$ DMSO) or kavalactones $(50 \mu \mathrm{M})$ or the KRE $(5 \mu \mathrm{g} / \mathrm{mL})$ treated LNCaP and C4-2B cells were carried out by following the kit instruction. HRP used the $\mathrm{H}_{2} \mathrm{O}_{2}$ byproduct in the MAO-A reaction to convert the non-fluorescence substrate resazurin into the fluorescence substrate resorufin, which 
was measured by a fluorescence spectrophotometer using the excitation value of $530 \mathrm{~nm}$ and the emission value of $585 \mathrm{~nm}$.

\section{Western blot analysis}

Protein lysates preparation and Western blotting analysis were performed according to our previously published paper ${ }^{[22]}$. Tubulin levels were used as a loading control.

\section{Immunohistochemistry}

Antigen retrieval method, titration of anti-human Ki67 (1:100) antibody, and immunohistochemistry staining of prostate tissues from control and the KRE containing food fed TRMAP mice were carried out by following our published papers ${ }^{[9,16]}$.

\section{Molecular docking studies ${ }^{[23]}$}

First, kawain and methysticin molecules were generated by Pymol software and converted to the pdbqt format using Open Babel. Then, the autodock 4 software from the Scripps Research Institute was used to dock kawain and methysticin with LSD1 protein (PDB code: pdb 2hkO) or MAO-A (pdb 2bxs) from MGLTools. Finally, Pymol was used to image the conformations of the molecule and protein interaction.

\section{Statistical analysis}

Analysis of variance or Student's $t$-test followed by the Bonferroni $t$-test for multiple comparisons was used to compared means of organ and body weights and food consumptions between vehicle control and KRE treatments over time. The Mann-Whitney $U$ and Kolmogorov-Smirnov test was used for GU weight comparison among different treatment groups. The comparisons of the percentages of mice with different pathologic stages or with palpable tumors among different treatment groups were tested by using the $\chi^{2}$ or Fisher exact test.

\section{RESULTS}

\section{Dietary feeding of the KRE for 6 weeks inhibits mouse HG-PIN and prostate adenocarcinoma in TRAMP mice}

To examine whether the KRE inhibits HG-PIN and prostate adenocarcinoma occurrence, TRAMP mice were given vehicle control, $0.3 \% \mathrm{KRE}$, or $0.6 \% \mathrm{KRE}$ containing food from 6 weeks of age to 12 weeks of age [Figure 1A]. Figure $1 \mathrm{~B}$ and $\mathrm{C}$ show that dietary feeding of $0.3 \%$ and $0.6 \%$ KRE inhibited HG-PIN by $43.5 \%$ and $59.7 \%$, respectively, and prostate adenocarcinomas by $53.5 \%$ and $66.4 \%$, respectively $(P<0.05$; Fisher exact test). These results suggest that the KRE has a cancer-preventive activity for early-stage prostate cancer.

\section{Dietary feeding of the KRE for 18 weeks reduced tumor burden in TRAMP mice}

To determine whether the KRE can affect tumor burdens at late stage of prostate cancer, TRAMP mice at 6 weeks of age were administrated with vehicle control, $0.3 \%$ or $0.6 \% \mathrm{KRE}$ containing food for 18 weeks. The mean GU weight of the mice fed with $0.6 \% \mathrm{KRE}$ containing food was significantly reduced when compared to the control group (Figure 2A; $1.98 \mathrm{~g} \pm 2.07 \mathrm{~g}$ vs. $3.63 \mathrm{~g} \pm 4.28 \mathrm{~g} ; P<0.05$; Mann-Whitney $U$ and Kolmogorov-Smirnov test). The percentages of large tumors ( $\mathrm{GU}$ weight $>0.9 \mathrm{~g}$ ) were also decreased from $86.4 \%$ in the control group to $52.2 \%$ and $43.5 \%$ in $0.3 \%$ and $0.6 \%$ KRE food groups, respectively [Figure $2 \mathrm{~B}$ ]. In addition, administration of $0.6 \%$ KRE containing food to TRAMP mice led to a decrease in food consumption, and both $0.3 \%$ and $0.6 \% \mathrm{KRE}$ food suppressed the body weight gain over time [Figure $2 \mathrm{C}$ and $\mathrm{D}$ ], as well as increased liver weight (data not shown) compared to control food. 
A
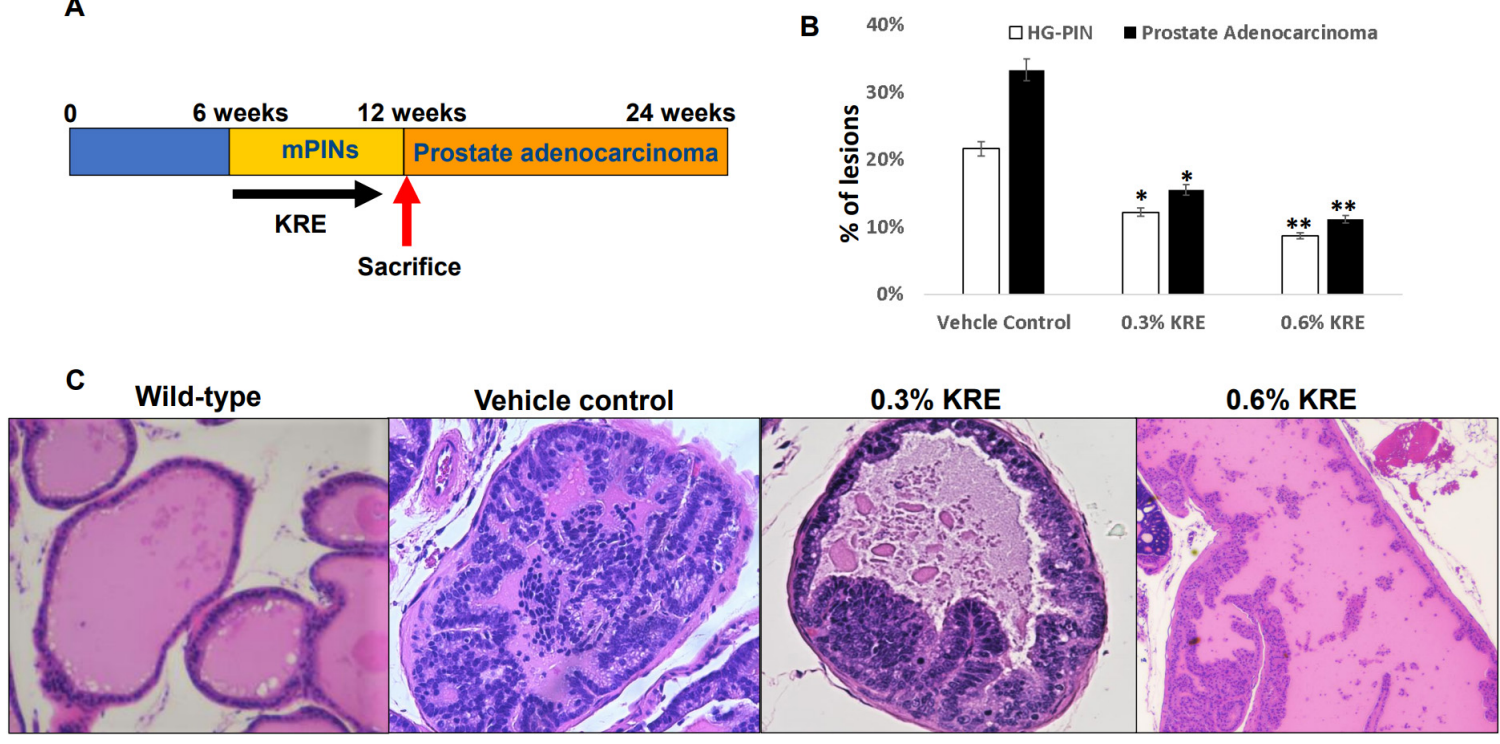

Figure 1. Dietary feeding of the KRE inhibits the occurence of HG-PIN and prostate adenocarcinoma in TRAMP mice. (A) A simple illustration of prevention protocol by the KRE in the TRAMP model. (B) Percentages of HG-PIN or adenocarcinoma lesions in the prostates of 12 -week-old, vehicle control vs. $0.3 \%$ or $0.6 \%$ KRE conatining food administrated to TRAMP mice. The prostates were from 8 mice in each group, and the total number of prostate acinus was counted. Fisher exact test show $P<0.05$. (C) Prostates in 12weeks-old, vehicle control vs. $0.3 \%$ or $0.6 \%$ KRE containing food fed TRAMP mice were examined by H\&E staining and histological evaluation. 200× magnifications of images are shown. KRE: Kava root extract; HG-PIN: high-grade prostatic intraepithelial neoplasia; TRAMP: transgenic adenocarcinoma of the mouse prostate. ${ }^{\star} P<0.05$ and ${ }^{\star \star} P<0.01$, respectively.

Kavalactones and KRE inhibit MAO-A activities in prostate cancer cell lines LNCaP and C4-2B cells Several studies reported that MAO-A was associated with aggressive prostate cancer and promoted prostate tumor growth and metastasis ${ }^{[20,21,24]}$. In addition, MAO-A is a key target for the treatment of neuropsychiatric and neurodegenerative disorder ${ }^{[19]}$. We therefore have examined whether the KRE and its active components kavalacotones can inhibit cellular MAO-A activity in LNCaP and C4-2B cells. First, we used the autodock program to dock kavalactones with MAO-A protein (PDB code: pdb 2bxs). Kawain and methysticin have predicted $\mathrm{IC}_{50}$ values of $3.85 \mu \mathrm{M}$ and $880.78 \mathrm{nM}$, respectively. Pymol program was used to observe the superimposed binding of kawain and methysticin to the flavin adenosine dinucleotide (FAD)binding and the active site of MAO-A and the kavalactones were predicted to form hydrogen bonds [Figure $3 \mathrm{~A}$ and $\mathrm{B}$ ].

In addition, the cellular MAO activities were examined by MAO-GLO assay after LNCaP and C4-2B cells were treated with KRE and kavalactones for $24 \mathrm{~h}$. Figure $3 \mathrm{C}$ and $\mathrm{D}$ show that kavalactones and $\mathrm{KRE}$ significantly inhibited cellular MAO activities by about $25 \%$ to $51 \%(P<0.05)$. These results suggest that kavalactones and KRE are weak inhibitors of MAO activity in prostate cancer cells.

\section{Kavalactones inhibit LSD1 activities in prostate cancer cells and dietary feeding of KRE is associated with reduced LSD1 activities in prostate tumors}

LSD1 is an epigenetic enzyme responsible for demethylation of $\mathrm{H}_{3} \mathrm{~K} 4$ and $\mathrm{H} 3 \mathrm{~K} 9$ for reprogramming gene expression profile and suggested to have a critical role in the progression of castration-resistant prostate cancer via interacting with $\mathrm{AR}^{[25,26]}$. Given the catalytic activity of both MAO-A and LSD1 resides in the amine oxidase domain and is dependent on its co-factor $F A D^{[18]}$, we therefore examined whether kavalactones could also affect LSD1 activity in prostate cancer. Molecular docking analysis revealed that kawain and methysticin superimpose FAD and binds to the FAD-binding domain of LSD 1 and interacts with important amino acids, methionine 332 and arginine 316, respectively [Figure $4 \mathrm{~A}$ and B]. 
A
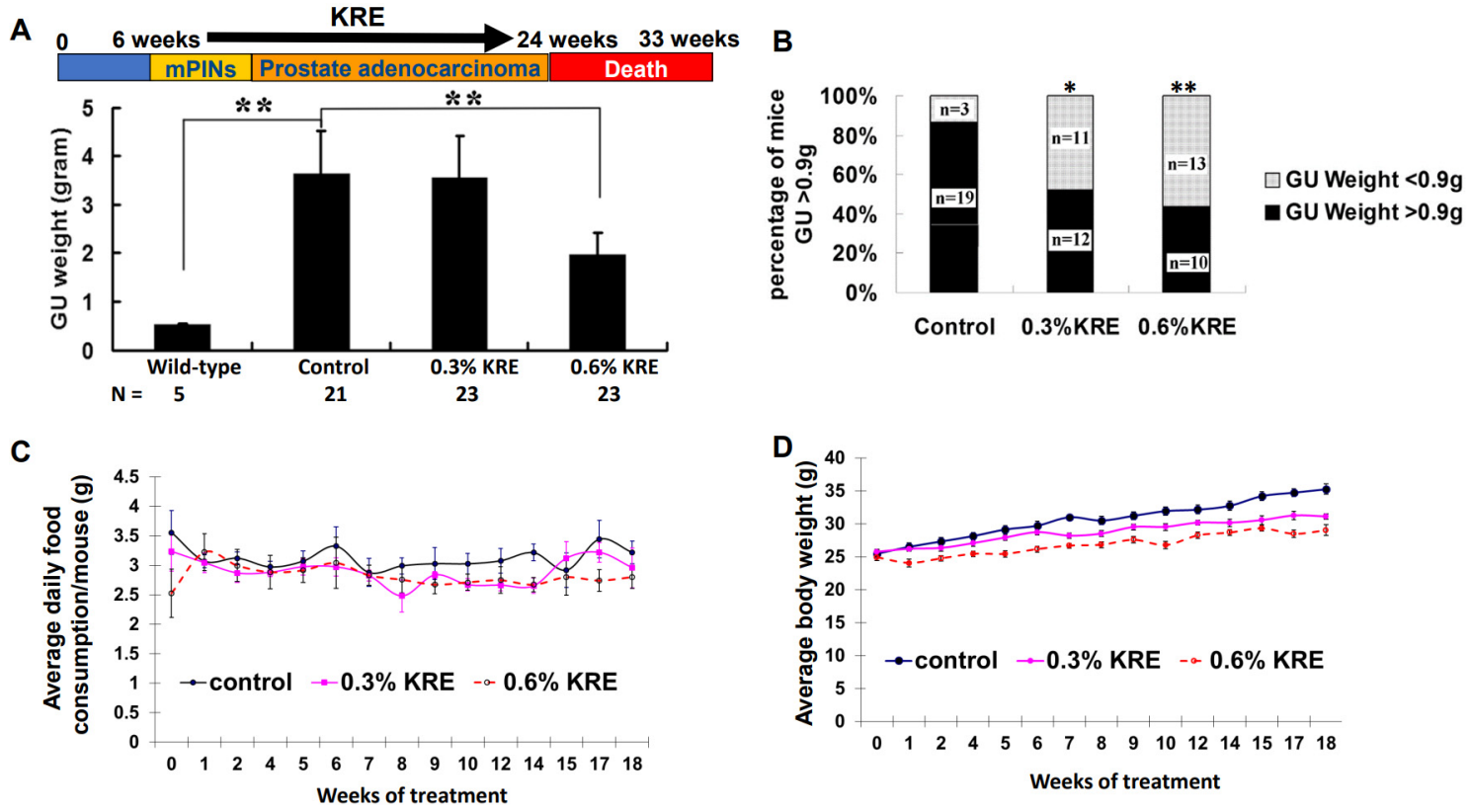

Figure 2. Dietary feeding of the KRE reduces tumorigenesis in TRAMP mice. (A) Upper panel, schematic presentation of intervention protocol by the KRE in the TRAMP model. Lower panel, average GU weights in different treatment groups. ${ }^{\star \star} P<0.01$. (B) Percentages of mice with large tumor formation. ${ }^{\star} P<0.05$ and ${ }^{\star \star} P<0.01$. (C) $0.6 \% \mathrm{KRE}$ food results in a reduction of food consumption over time. (D) Dietary feeding of $0.3 \%$ and $0.6 \% \mathrm{KRE}$ food reduces mean body weight gain of TRAMP mice over time. KRE: Kava root extract; TRAMP: transgenic adenocarcinoma of the mouse prostate; GU: genitourinary.

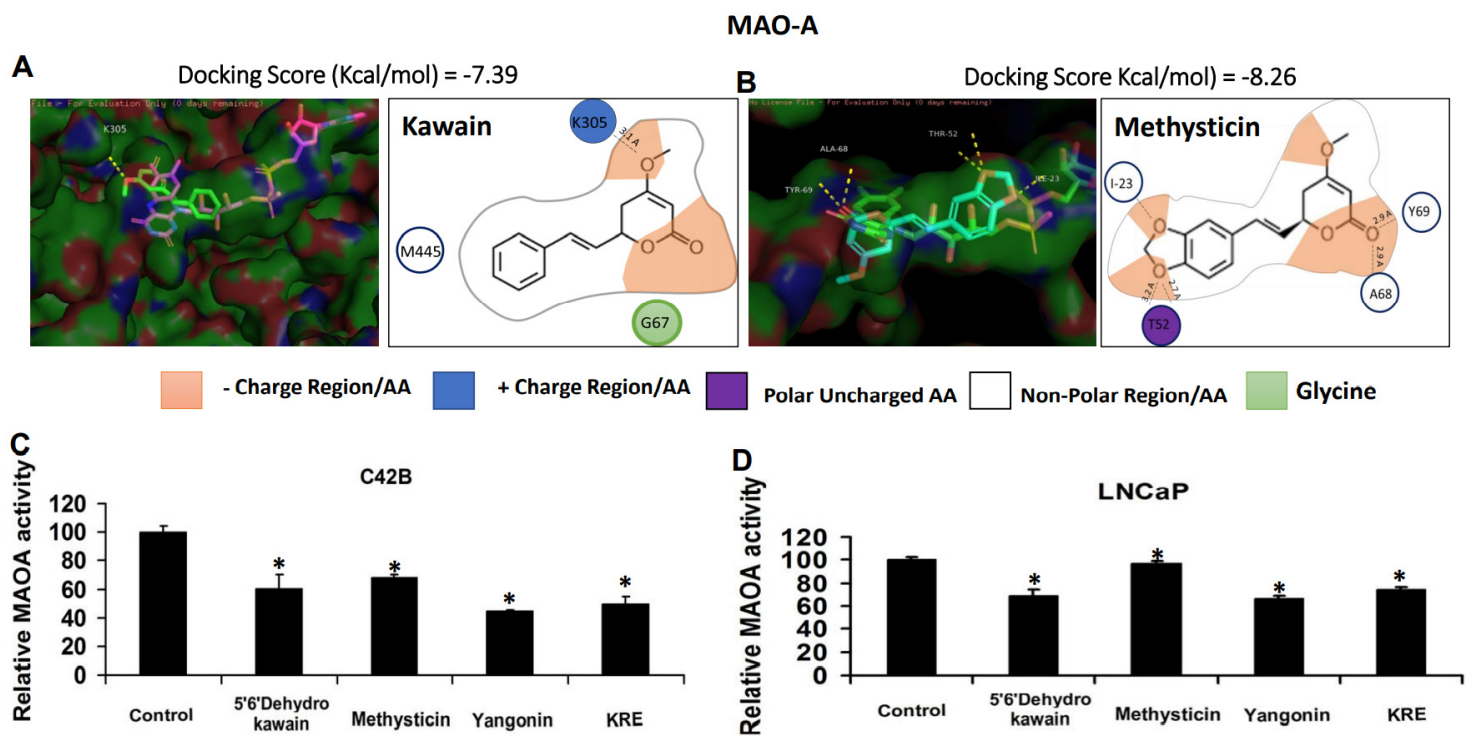

Figure 3. Kavalactones inhibits MAO-A enzyme activity. (A, B) Kawain and methysticin were docked with MAO-A protein by Autodocktools program, and the superimposed binding of kawain and methysticin to MAO-A, respectively, was observed in Pymol program. 2D molecular structures of kawain and methysticin are shown to interact with key amino acids on the predicted binding site. (C) MAO-A enzyme activities were analyz ed in prostate cancer C4-2B and LNCaP cell lysates after treatment with $0.1 \%$ DMSO (control), KRE and kavalactones for 24 h. MAO-A: Monoamine oxidase A; KRE: kava root extract. ${ }^{\star} P<0.05$.

In vitro LSD 1 inhibitory activity assay shows that kawain and methysticin have the most significant inhibition against LSD 1 enzyme activity among the tested kavalactones [Figure $4 \mathrm{C}$ ]. In addition, protein expression levels of dimethylated $\mathrm{H} 3 \mathrm{~K} 9$ were observed to be significantly increased in KRE, methysticin, 


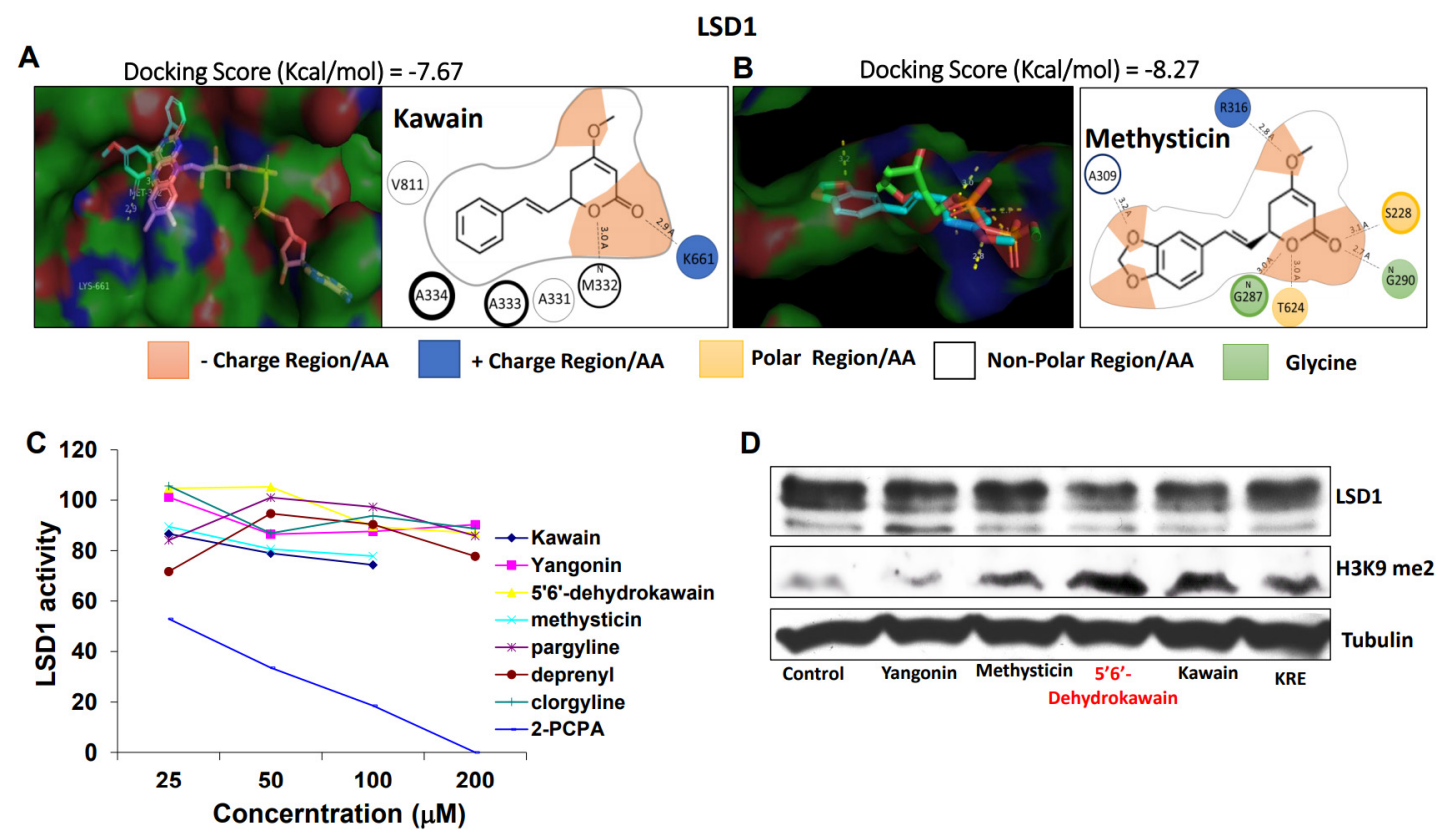

Figure 4. Kavalactones inhibits LSD1 enzyme activity. (A, B) Autodocktools program was used to dock kawain and methysticin with LSD1 protein, and Pymol program was used to observe the superimposed binding of kawain and methysticin to LSD1, respectively. 2D molecular structures of kawain and methysticin are shown to interact with key amino acids within the predicted binding site. (C) In vitro LSD1 enzyme activities were analyzed using LSD1 inhibitor screening kit. (D) Western blotting analysis of LSD1 expression and H3K9 dimethylation. LSD1: Lysine-specific demethylase 1.

5',6'-dehydrokawain, or kawain treated LNCaP cells without affecting LSD1 expression [Figure 4D]. Our previous studies have also reported that KRE, methysticin and kawain inhibited the gene transcription of PSA and TMPRSS2, two AR target genes. These results together suggest that KRE, methysticin, and kawain reduce LSD1 enzyme activity in cells leading to inhibition of AR signaling.

\section{KRE containing food increased H3K9 dimethylation and reduced Ki67 expression in tumor tissues} Similar to the in vitro findings as described above, Western blotting analysis confirmed that KRE containing food increased $\mathrm{H} 3 \mathrm{~K} 9$ dimethylation without obvious effects on LSD1 expression in prostate tissues of the TRAMP mice [Figure 5A] and tumor tissues of a prostate cancer PDX model from our previous studies [Figure 5B]. In addition, immunohistochemistry analysis revealed that the number of Ki67-positive cells in the prostate of KRE-fed TRAMP mice was $26.3 \% \pm 4.7 \%$ compared with $49.1 \% \pm 10.2 \%$ in those from the vehicle control treatment $(P<0.05$; Figure $5 \mathrm{C}$ and $\mathrm{D})$. This finding suggests that KRE feeding inhibited LSD1 activity, leading to the anti-proliferative effects of KRE on prostate tumor tissues of mouse models and therefore delaying the progression of prostate cancer.

\section{DISCUSSION}

Although kavalactones have been suggested to be responsible at least in part for both the anxiolytic effect and the anti-prostate cancer effect, the molecular targets of kavalactones remain understudied. In this study, our results have shown that kawain and methysticin are weeks but cell-active inhibitors for both MAO-A and LSD1 enzymes. Therefore, our study has provided the first structure base for further developing more potent dual inhibitors of MAO-A and LSD 1 through chemical modification of the kavalactone skeleton. Given both MAO-A and LSD1 have been demonstrated to be critically involved in prostate cancer progression. Dual inhibition of MAO-A and LSD1 may have significant translation values in prostate cancer prevention and treatment. 
A

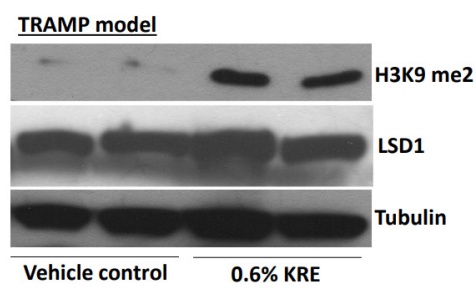

B

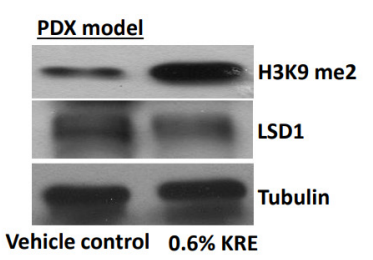

c Control

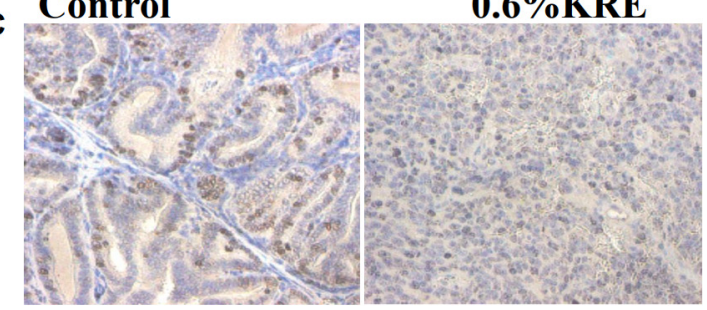

D

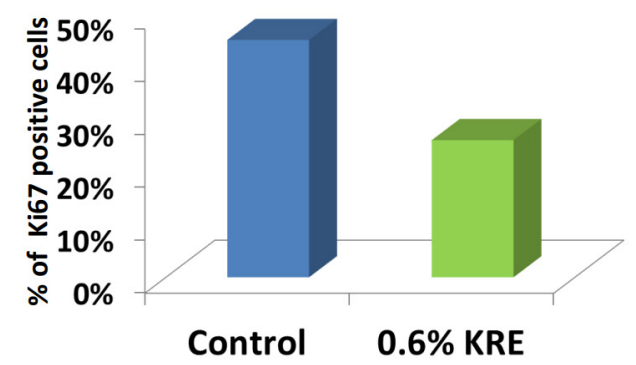

Figure 5. Dietary feeding of KRE enhances H3K9 dimethylation and inhibits cell proliferation in vivo. (A, B) Western blot analysis of H3K9 dimethylation in mouse dorsolateral prostates of TRAMP mice and prostate cancer PDX tumors, respectively, in vehicle control and $0.6 \%$ KRE treatment groups. (C) Ki67 immunohistochemistry staining was shown in the prostates of TRAMP mice that was treated with vehicle control vs. $0.6 \% \mathrm{KRE}$ containing food for 18 weeks. $200 \times$ magnifications of images are presented. (D) The percentage of positive stained cells was calculated after 20 fields from ten mouse prostates were counted in each group. KRE: Kava root extract; H3K9: histone H3 lysine 9; TRAMP: transgenic adenocarcinoma of the mouse prostate; PDX: patient-derived xenograft; LSD1: lysinespecific demethylase 1.

LSD1 was reported to interact with the AR and serves as a co-factor to regulate the transcriptional expression of AR target genes ${ }^{[25,26]}$. Our previous studies demonstrated that kavalactones inhibited mRNA expression of PSA and TMPRESS2, two key AR target genes, in LNCaP cells and tumor tissues of prostate cancer PDX model ${ }^{[9]}$. Whether the inhibitory effect of kawain and methysticin on AR signaling is dependent, at least in part, on LSD1 expression warrants further studies. These studies will facilitate further understanding of molecular mechanisms by which these kavalactones regulate AR transcription.

Previous studies by our group and others have demonstrated that kava chalcones (flavokawain A) and a kavalactone-rich kava fraction B (free of flavokawains), respectively, are effective in preventing the occurrences of HG-PIN and early prostate adenocarcinoma and reducing tumor burdens in the TRAMP model ${ }^{[10,16]}$. In addition, we have shown that administration of flavokawain A containing food to TRAMP mice resulted in the eradication of distant organ metastasis ${ }^{[16]}$, whereas a kavalactone-rich kava fraction $\mathrm{B}$ was shown to significantly decrease the incidences of neuroendocrine carcinoma in TRAMP mice by absolute $42.9 \%^{[10]}$. The KRE used here contains both kavalactones and flavokawains, including $2.7 \%$ kawain, $1.4 \%$ methysticin, 1.75\% 5,6-dehydrokawain, 3.08\% Yangonin, 0.33\% flavokawain B, and $0.21 \%$ flavokawain $\mathrm{A}^{[9]}$. The KRE may be able to target different stages and heterogenity of prostate cancer. Consistent with the previous studies, the KRE has similar potency as a kavalactone-rich kava fraction B for its chemopreventive effect in prostate cancer in the TRAMP model. However, further studies are still needed to investigate whether there are significant synergistic or antagonistic effects between flavokawains and kavalactones on prostate cancer.

Dietary feeding of the KRE used here resulted in decreased body weight gain and liver enlargement (data not shown). This result is also consistent with the report by Tang et al. ${ }^{[10]}$ that the kavalactone-rich kava fraction B (free of flavokawains) suppressed body weight gain and induced liver enlargement 
(hepatomegaly) without noticeable changes in liver function. In contrast, the changes in body weight gain and organ weight after long-term dietary feeding of flavokawain A in TRAMP mice have not been observed in our previous studies ${ }^{[16]}$. Therefore, it is unlikely that flavokawain A is responsible for the reported "kava hepatotoxicity". Flavokawain A could be a safer product for cancer prevention than the KRE and the kavalactone-rich kava fraction B.

In summary, the KRE product containing both kavalactones and flavokawains reduced the incidence of HG-PIN and early-stage prostate adenocarcinoma and inhibited tumorigenesis. The KRE and kavalactone weakly inhibited both MAO-A and LSD1 activities in prostate cancer, leading to their inhibitory effect on AR signaling and cell proliferation. This study has provided a new knowledge basis for developing more effective dual inhibitors of MAO-A and LSD1 enzymes for prostate cancer prevention and treatment.

\section{DECLARATIONS}

\section{Acknowledgments}

This work was supported in part by NIH awards 1R01CA193967-01A1 and DOD/Prostate Cancer Research Program PC100869, and DOD/Prostate Cancer Research PC121803 (to Zi X).

\section{Authors' contributions}

Conceived and designed study: Uchio E, Zi X

Analysed and interpreted data: Uchio E, Zi X

Drafted and revised the manuscript: Uchio E, Zi X

Performed experiments and the statistical analyses: Li X, Song L, Xu S, Tippin M, Meng S, Xie J

All authors read and approved the manuscript.

\section{Availabilities of data and materials}

The data that support the findings of this study are available from the corresponding author upon reasonable request.

\section{Financial support and sponsorship}

This work was supported in part by NIH awards 1R01CA193967-01A1 and DOD/Prostate Cancer Research Program PC100869, and DOD/Prostate Cancer Research PC121803 (to Zi X).

\section{Conflicts of interest}

All authors declared that there are no conflicts of interest.

\section{Ethical approval and consent to participate}

The animal experiments were performed according to the approved protocol (\#2007-2740) by University of California, Irvine, Institutional Animal Care and Use Committee.

\section{Consent for publication}

Not applicable.

\section{Copyright}

(c) The Author(s) 2021.

\section{REFERENCES}

1. Key Statistics for Prostate Cancer. Available from: https://www.cancer.org/cancer/prostate-cancer/about/key-statistics.html. [Last accessed on 3 Jun 2021].

2. Carlsson S, Benfante N, Alvim R, et al. Long-term outcomes of active surveillance for prostate cancer: the memorial sloan kettering cancer center experience. J Urol 2020;203:1122-7. DOI PubMed PMC 
3. Gregg JR, Zhang X, Chapin BF, et al. Adherence to the Mediterranean diet and grade group progression in localized prostate cancer: an active surveillance cohort. Cancer 2021;127:720-8. DOI PubMed

4. $\quad$ Singh YN. Kava: an overview. J Ethnopharmacol 1992;37:13-45. DOI PubMed

5. Sarris J, Byrne GJ, Bousman CA, et al. Kava for generalised anxiety disorder: A 16-week double-blind, randomised, placebocontrolled study. Aust N Z J Psychiatry 2020;54:288-97. DOI PubMed

6. Savage KM, Stough CK, Byrne GJ, et al. Kava for the treatment of generalised anxiety disorder (K-GAD): study protocol for a randomised controlled trial. Trials 2015;16:493. DOI PubMed PMC

7. Smith K, Leiras C. The effectiveness and safety of Kava Kava for treating anxiety symptoms: a systematic review and analysis of randomized clinical trials. Complement Ther Clin Pract 2018;33:107-17. DOI PubMed

8. Steiner GG. The correlation between cancer incidence and kava consumption. Hawaii Med J 2000;59:420-2. PubMed

9. Li X, Liu Z, Xu X, et al. Kava components down-regulate expression of AR and AR splice variants and reduce growth in patientderived prostate cancer xenografts in mice. PLoS One 2012;7:e31213. DOI PubMed PMC

10. Tang SN, Zhang J, Jiang P, et al. Gene expression signatures associated with suppression of TRAMP prostate carcinogenesis by a kavalactone-rich kava fraction. Mol Carcinog 2016;55:2291-303. DOI PubMed PMC

11. Liu Z, Xu X, Li X, et al. Kava chalcone, flavokawain A, inhibits urothelial tumorigenesis in the UPII-SV40T transgenic mouse model. Cancer Prev Res (Phila) 2013;6:1365-75. DOI PubMed PMC

12. Leitzman P, Narayanapillai SC, Balbo S, et al. Kava blocks 4-(methylnitrosamino)-1-(3-pyridyl)-1-butanone-induced lung tumorigenesis in association with reducing O6-methylguanine DNA adduct in A/J mice. Cancer Prev Res (Phila) 2014;7:86-96. DOI PubMed PMC

13. Supramaniam R, O'Connell D, Tracey E, Sitas F. Cancer in New South Wales Migrants 1991 to 2001. Available from: https://www.cancercouncil.com.au/wp-content/uploads/2010/09/A-Pg-1-23-Intro.pdf. [Last accessed on 3 Jun 2021].

14. Shibuya K, Mathers CD, Boschi-Pinto C, Lopez AD, Murray CJ. Global and regional estimates of cancer mortality and incidence by site: II. Results for the global burden of disease 2000. BMC Cancer 2002;2:37. DOI PubMed PMC

15. Li X, Pham V, Tippin M, et al. Flavokawain B targets protein neddylation for enhancing the anti-prostate cancer effect of Bortezomib via Skp2 degradation. Cell Commun Signal 2019;17:25. DOI PubMed PMC

16. Li X, Yokoyama NN, Zhang S, et al. Flavokawain A induces deNEDDylation and Skp2 degradation leading to inhibition of tumorigenesis and cancer progression in the TRAMP transgenic mouse model. Oncotarget 2015;6:41809-24. DOI PubMed PMC

17. Shappell SB, Thomas GV, Roberts RL, et al. Prostate pathology of genetically engineered mice: definitions and classification. The consensus report from the Bar Harbor meeting of the Mouse Models of Human Cancer Consortium Prostate Pathology Committee. Cancer Res 2004;64:2270-305. DOI PubMed

18. Zheng YC, Duan YC, Ma JL, et al. Triazole-dithiocarbamate based selective lysine specific demethylase 1 (LSD1) inactivators inhibit gastric cancer cell growth, invasion, and migration. J Med Chem 2013;56:8543-60. DOI PubMed PMC

19. Prinsloo D, van Dyk S, Petzer A, Petzer JP. Monoamine oxidase inhibition by kavalactones from kava (piper methysticum). Planta Med 2019;85:1136-42. DOI PubMed

20. Wang K, Luo J, Yeh S, et al. The MAO inhibitors phenelzine and clorgyline revert enzalutamide resistance in castration resistant prostate cancer. Nat Commun 2020;11:2689. DOI PubMed PMC

21. Wu JB, Shao C, Li X, et al. Monoamine oxidase A mediates prostate tumorigenesis and cancer metastasis. J Clin Invest 2014;124:2891-908. DOI PubMed PMC

22. Zi X, Simoneau AR. Flavokawain A, a novel chalcone from kava extract, induces apoptosis in bladder cancer cells by involvement of Bax protein-dependent and mitochondria-dependent apoptotic pathway and suppresses tumor growth in mice. Cancer Res 2005;65:3479-86. DOI PubMed

23. Pham V, Rendon R, Le VX, et al. Gartanin is a novel NEDDylation inhibitor for induction of Skp2 degradation, FBXW2 expression, and autophagy. Mol Carcinog 2020;59:193-201. DOI PubMed PMC

24. Zhao H, Nolley R, Chen Z, Reese SW, Peehl DM. Inhibition of monoamine oxidase A promotes secretory differentiation in basal prostatic epithelial cells. Differentiation 2008;76:820-30. DOI PubMed PMC

25. Sehrawat A, Gao L, Wang Y, et al. LSD1 activates a lethal prostate cancer gene network independently of its demethylase function. Proc Natl Acad Sci U S A 2018;115:E4179-88. DOI PubMed PMC

26. Metzger E, Wissmann M, Yin N, et al. LSD1 demethylates repressive histone marks to promote androgen-receptor-dependent transcription. Nature 2005;437:436-9. DOI PubMed 